\title{
НЕРАСПЛЫВАЮЩИИСЯ ВОЛНОВОЙ ПАКЕТ В ЛИНЕЙНОМ ГАРМОНИЧЕСКОМ ОСЦИЛЛЯТОРЕ
}

\section{Введение}

В [ $\left.{ }^{1}\right]$ показано, что нерелятивистское уравнение Шредингера свободной частицы имеет решение, изображающее движущийся с постоянным ускорением нерасплывающийся волновой пакет. В настоящей статье мы рассмотрим аналогичную задачу для связанной частицы. Покажем, что в одномерном случае нерасплывающийся волновой пакет возможен только в гармоническом осцилляторе, и найдем все описывающие его основные формулы.

\section{Решение уравнения Шредингера}

Пусть частица массы $m$ движется в одномерном потенциальном поле $V(x)$. Будем искать решение уравнения Шредингера

$$
\left(\hbar^{2} / 2 m\right) \partial^{2} \psi / \partial x^{2}+i \hbar \partial \psi / \partial t-V \psi=0
$$

в виде

$$
\psi=A(f(x, t)) \exp (i g(x, t))
$$

с вещественными функциями $A$ и $g$. Подстановка этого выражения в уравнение (1) дает по разделении вещественной и мнимой частей два уравнения

и

$$
f_{x}^{2} A^{\prime \prime}+f_{x x} A^{\prime}-\left[g_{x}^{2}+2 m \hbar^{-1} g_{t}+2 m \hbar^{-2} V\right] A=0
$$

$$
2\left[f_{x} g_{x}+m \hbar^{-1} f_{t}\right] A^{\prime}+g_{x x} A=0,
$$

где индексами у $f$ и $g$ обозначены частные производные по $x$ и $t$, a штрих у $A$ означает производную по $f$.

Положив

$$
d f=f_{x} d x+f_{t} d t=0,
$$

находим

$$
v \equiv d x / d t=-f_{t} / f_{x},
$$

где через $v$ обозначена скорость движения точки, в которой $f$ и, следовательно, $A$ имеют фиксированное значение. В уравнении (3), по разделении его на $f_{x}^{2}$, оба коэффициента могут зависеть от $x$ и $t$ только через $f$. Так, 


$$
d\left(f_{x x} f_{x}^{-2}\right)=0, \quad \text { если } \quad d f=0 .
$$

Отсюда найдем характер зависимости $v$ от $x$ и $t$. Вычисляя дифференциал, заменяя в нем $d x$ на $v d t$ и сокращая на $f_{x}{ }^{-3} d t$, получаем

$$
v\left(f_{x x x} f_{x}-2 f_{x x}\right)+f_{x x t} f_{x}-2 f_{x x} f_{x t}=0 .
$$

Беря производные по $x$ от равенства $f_{t}=-v f_{x}$, находим

$$
f_{x t}=-v f_{x x}-v_{x} f_{x}
$$

и

$$
f_{x x t}=-v f_{x x x}-2 v_{x} f_{x x}-v_{x x} f_{x} .
$$

Подставляя эти выражения в равенство (8), находим

$$
v_{x x} f_{x}^{2}=0
$$

откуда, поскольку $f_{x} \neq 0$, следует

$$
v_{x x}=0 .
$$

Этот результат показывает, что скорость перемещения фиксированного значения мөдуля волновой функции может зависеть от координаты только линейно. Но так как мы ищем решение в виде нерасплывающегося волнового пакета, то $v$ вообще не должна зависеть от координаты, а может зависеть только от времени. Итак,

$$
f_{t} / f_{x}=-v(t) \text {. }
$$

Обратимся теперь вновь к уравнениям (3) и (4). Так как (3) уравнение второго порядка для $A$, то удовлетворить уравнению первого порядка (4) возможно только приравняв коэффициенты нулю. Точнее, равенство нулю первого коэффициента влечет за собой равенство нулю и второго. В самом деле, если

$$
f_{x} g_{x}+(m / \hbar) f_{t}=0,
$$

то, в силу (13),

$$
g_{x}=m v(t) / \hbar,
$$

откуда

$$
g_{x x}=0
$$

Интегрируя (15), находим

$$
g=m v(t) x / \hbar+\eta(t) .
$$

Подставляя это выражение в уравнение (3), получаем его в виде

$$
A^{\prime \prime}+f_{x x} f_{x}^{-2} A^{\prime}-2 m \hbar-2 f_{x}^{-2}\left(V+m \dot{v} x+m v^{2} / 2+\dot{\hbar} \eta\right) A=0,
$$

где точка означает производную по времени.

Обозначим временно

$$
f_{x x} f_{x}^{-2}=X(f)
$$

Интегрируя это соотношение, получаем

$$
f_{x}=C(t) \exp \left(\int X d f\right) .
$$


Вторичное интегрирование дает

$$
x=\gamma(t)+C^{-1}(t) \int \exp \left(-\int^{f} X(\varphi) d \varphi\right) d f .
$$

Далее покажем, что $C$ фактически не зависит от $t$. Для этого перепишем формулу (21) в виде

$$
C(t)(x-\gamma)=\int \exp \left(-\int^{f} X(\varphi) d \varphi\right) d f
$$

и возьмем производную по времени

$$
\dot{C}(x-\gamma)-C \dot{\gamma}=f_{t} \exp \left(-\int X d f\right)
$$

нли

$$
f_{t}=[\dot{C}(x-\gamma)-\dot{C}] \exp \left(\int X d f\right) .
$$

Отсюда и из формул (13) и (20) следует

$$
\dot{C}(x-\gamma)-\dot{\gamma}+C v=0 .
$$

Так как здесь все члены, кроме $\dot{C} x$, зависят только от времени, то $\dot{C} x=0$, откуда $C=$ const. Перепишем теперь формулы (20), (22) и (25) в виде:

$$
\begin{gathered}
f_{x}=C \exp \left(\int X d f\right) \\
C(x-\gamma)=\int \exp \left(-\int^{f} X(\varphi) d \varphi\right) d f
\end{gathered}
$$

H

$$
\dot{\gamma}=v \text {. }
$$

Далее примем в уравнении (18) в качестве нового аргумента вместо $f$, согласно формуле (27), величину

$$
u=x-\gamma
$$

и подставим в последний член вместо $f_{x}$ выражение (26). Тогда получим

$$
d^{2} A / d u^{2}-2 m \hbar^{-2}\left(V+m \dot{v} x+m v^{2} / 2+\hbar \dot{\eta}\right) A=0 .
$$

Введя $u$ вместо $x$, перепишем уравнение в виде

$$
d^{2} A / d u^{2}-2 m \dot{\imath}^{-2}\left[V(u+\gamma)+m \dot{v} u+m \dot{v} \gamma+m v^{2} / 2+\dot{\hbar} \dot{\eta}\right] A=0,
$$

где $u+\gamma$ в скобках после $V$ означает аргумент.

Согласно формуле (27) новый аргумент $u$ однозначно связан с прежним аргументом $f$, от которого $A$ только и зависит. Отсюда следует, что коэффициент при $A$ в уравнении (31) может зависеть от времени только через $u$. Производная его, взятая по времени помимо $u$, должна равняться нулю:

$$
V^{\prime}(u+\gamma) \dot{\gamma}+m \ddot{v} u+m \ddot{v} \gamma+m \dot{v} \dot{\gamma}+m v \dot{v}+\ddot{\hbar} \ddot{\eta}=0 .
$$

Отсюда с учетом формулы (28) следует

$$
V^{\prime}(u+\gamma)=-(m \ddot{v} / v)(u+\gamma)-2 m \dot{v}-\hbar \ddot{\eta} / v .
$$

Интегрируя по аргументу $u+\gamma$ и заменяя его вновь на $x$, получаем 


$$
V(x)=-(m \ddot{v} / v)\left(x^{2} / 2\right),
$$

где линейный и постоянный члены опущены (мы принимаем, как обычно, центр сил за начало координат). Так как частица связана, то коэффициент - $m \ddot{v} / v$ должен быть положительным; полагая

$$
\ddot{v} / v=-\omega^{2},
$$

находим окончательно

$$
V(x)=m \omega^{2} x^{2} / 2 .
$$

Итак, одномерный нерасплывающийся волновой пакет возможен для связанной частицы только в гармоническом осцилляторе.

Подставляя выражение (36) потенциала в уравнение (31), находим

$$
\begin{gathered}
d^{2} A / d u^{2}-2 m \hbar^{-2}\left[m \omega^{2} u^{2} / 2+m\left(\omega^{2} \gamma+\dot{v}\right) u+\right. \\
\left.+\left(m \omega^{2} \gamma^{2} / 2+m \dot{v} \gamma+m v^{2} / 2+\dot{\hbar} \dot{\eta}\right)\right] A=0 .
\end{gathered}
$$

Здесь коэффициент при $A$ может зависеть только от $u$; следовательно, приравнивая коэффициент при $u$ и свободный член постоянным, имеем

$$
\omega^{2} \gamma+\dot{v}=C_{1} \omega^{2}
$$

и

$$
\hbar^{-1} m\left(\omega^{2} \gamma^{2} / 2+v^{2} / 2+\dot{v} \gamma\right)+\dot{\eta}=C_{2},
$$

после чего уравнение (37) принимает вид

$$
d^{2} A / d u^{2}-2 m \hbar^{-2}\left[m \omega^{2} u^{2} / 2+m \omega^{2} C_{1} u+\hbar C_{2}\right] A=0 .
$$

Система уравнений (28), (35), (38) и (39) определяет функции времени $v, \gamma$ и $\eta$. Решая ее, находим:

$$
\begin{gathered}
v=K \omega \cos \omega t \\
\gamma=K \sin \omega t+C_{1}
\end{gathered}
$$

и

$$
\eta=C_{3}+\left(C_{2}-m \omega^{2} C_{1}^{2} / 2 \hbar\right) t-\left(K^{2} m \omega / 4 \hbar\right) \sin 2 \omega t,
$$

где $K$ и $C_{3}-$ постоянные интегрирования.

Нам осталось решить уравнение (40). Положив

$$
y=u+C_{1}=x-\gamma+C_{1}=x-K \sin \omega t,
$$

получим его в виде

$$
d^{2} A / d y^{2}-\left[m^{2}\left(\omega^{2} y^{2} / \hbar^{2}-\left(C_{1}^{2} m^{2} \omega^{2} / \hbar^{2}-2 C_{2} m \hbar^{-1}\right)\right] A=0 .\right.
$$

Это уравнение совпадает по форме с уравнением Шредингера для стационарного состояния гармонического осциллятора

$$
d^{2} \psi_{n} / d x^{2}-\left(m^{2} \omega^{2} x^{2} / \hbar^{2}-2 m E_{n} / \hbar^{2}\right) \psi_{n}=0,
$$

решение которого, удовлетворяющее граничному условию на бесконечности, имеет, как известно, вид

$$
\begin{gathered}
\psi_{n}(x, t)=\left(m_{\omega} / \hbar \pi\right)^{1 / 4}\left(2^{n} n !\right)^{-1 / 2} H_{n}\left(x\left(m_{\omega} / \hbar\right)^{1 / 2}\right) \times \\
\times \exp \left(-m_{\omega} x^{2} / 2 \hbar\right) \exp (-i(n+1 / 2) \omega t),
\end{gathered}
$$


где $n-$ неотрицательное целое число, $H_{n}-$ полином Әрмита, причем

$$
E_{n}=\hbar \omega(n+1 / 2) \text {. }
$$

Поскольку решение уравнения (45) тоже должно-удовлетворять граничному условию, то должно быть

$$
C_{1}^{2} m_{\omega^{2}}^{2} / 2 \hbar-C_{2}=\omega(n+1 / 2),
$$

и тогда, учитывая формулу (44), имеем

$$
\begin{gathered}
A \equiv A_{n}=(m \omega / \hbar \pi)^{1 / 4}\left(2^{n} n !\right)^{-1 / 2} H_{n}\left((m \omega / \hbar)^{1 / 2}(x-K \sin \omega t)\right) \times \\
\times \exp \left(-(m \omega / 2 \hbar)(x-K \sin \omega t)^{2}\right) .
\end{gathered}
$$

Формула (43) принимает более простой вид

$$
\eta=C_{3}-\omega t(n+1 / 2)-\left(K^{2} m \omega / 4 \hbar\right) \sin 2 \omega t .
$$

Осталось найти фазу. Для нее имеем формулу (17). Подставляя туда выражения (41) и (51) для $v$ и $\eta$ и отбрасывая несущественную для фазы постоянную $C_{3}$, находим

$$
g(x, t)=-(n+1 / 2) \omega t+K m \omega \hbar^{-1} \cos \omega t(x-(K / 2) \sin \omega t) .
$$

Итак, нестационарное решение уравнения Шредингера

$$
\partial^{2} \psi / \partial x^{2}+2 i m \hbar^{-1} \partial \psi / \partial t-\left(m \omega^{2} x^{2} / 2\right) \psi=0
$$

для линейного гармонического осциллятора, изображающее нерасплывающийся волновой пакет, имеет вид

$$
\begin{gathered}
\psi_{n K}(x, t)=(m \omega / \hbar \pi)^{1 / 4}\left(2^{n} n !\right)^{-1 / 2} H_{n}\left((m \omega / \hbar)^{1 / 2}(x-K \sin \omega t)\right) \times \\
\times \exp \left(-(m \omega / 2 \hbar)(x-K \sin \omega t)^{2}\right) \exp [-i(n+1 / 2) \omega t+ \\
\left.+i K m \omega \hbar^{-1} \cos \omega t(x-(K / 2) \sin \omega t)\right] .
\end{gathered}
$$

Иначе, пользуясь обозначением $\psi_{n}$ для стационарных состояний, можно написать

$$
\begin{gathered}
\psi_{n K}(x, t)=\psi_{n}(x-K \sin \omega t, t) \times \\
\times \exp \left[i K m \omega \hbar^{-1} \cos \omega t(x-(K / 2) \sin \omega t)\right] .
\end{gathered}
$$

Каждое такое нестационарное состояние соответствует некоторому стационарному состоянию, но отличается от него тем, что волновая функция совершает, не меняя формы, около центра гармонические колебания с частотой $\omega$ и произвольной амплитудой $K$. В литературе рассматривалось до сих пор, по-видимому, подобное состояние только для $n=0$ (см. $\left[{ }^{2}\right]$, с. $\left.87-89\right)$.

\section{Средняя энергия и разложение по стационарным состояниям}

Для более полной характеристики состояния $\psi_{n \kappa}$ вычислим среднюю энергию в этом состоянии и разложим его по стационарным состояниям. Не останавливаясь на деталях вычисления, приведем конечные результаты. Средняя энергия, вычисляемая по формуле

$$
\langle E\rangle_{n K}=\int_{-\infty}^{+\infty} \psi_{n K}^{*} i \hbar\left(\partial \psi_{n K} / \partial t\right) d x,
$$


оказывается равной

$$
\langle E\rangle_{n K}=(n+1 / 2) \hbar \omega+m K^{2} \omega^{2} / 2,
$$

т. е. является суммой энергии стационарного состояния $\psi_{n}$ и классической энергии частицы, осциллирующей с амплитудой $K$.

Разложение по стационарным состояниям имеет вид

$$
\psi_{n K}=\sum_{p=0}^{\infty} A_{n p}^{(K)} \psi_{p} .
$$

Вычисляя отсюда коэффициенты $A_{n p}^{(K)}$ по формуле

$$
A_{n p}^{(K)}=\int_{-\infty}^{+\infty} \psi_{p}^{*} \psi_{n K} d x
$$

находим

$$
A_{n p}^{(K)}=i^{n+p}(n ! p !)^{-1 / 2} \exp \left(-S^{2} / 2\right) Q_{n p}(S),
$$

где

$$
S=K(m \omega / 2 \hbar)^{1 / 2}
$$

и

$$
Q_{n p}(S)=\sum_{k=0}^{n} \frac{(-1)^{k} n ! p ! S^{n+p-2 k}}{k !(n-k) !(p-k) !} .
$$

Заметим, что $S^{2}$ равно отношению энергии колебания пакета к энергии кванта.

\section{Л ИТ Е Р А Т У Р А}

1. B erry, M. V., B a 1 a z s, N. L., Amer. J. Phys., 47, № 3, 264-267 (1979).

2. Ш и фф Л., Квантовая механика, М., ИИЛ, 1959.

Тартуский государственный университет
Поступила в редакцию 1/XII 1980

\section{P. KARD}

\section{MITTEHAJUV LAINEPAKETT LINEAARSES HARMOONILISES OSTSILLAATORIS}

Seotud osakest kirjeldaval ühemõōtmelisel Schrödingeri võrrandil on olemas mittehajuvaid lainepakette kujutavad mittestatsionaarsed lahendid ainult harmoonilise ostsillaatori juhul. Iga niisugune pakett on samasuguse kujuga nagu statsionaarses olekus, kuid ta vōngub mingi meelevaldse amplituudiga ostsillaatori põhisagedusel (valem (55), kus $\psi_{n}$ on statsionaarse oleku lainefunktsioon). Energia keskväärtus avaldub sellises mittestatsionaarses olekus valemiga (57) ja statsionaarsete olekute järgi arenduse (58) kordajad avalduvad valemitega $(60)-(62)$. 


\section{P. KARD}

\section{NONSPREADING WAVE PACKET IN THE LINEAR HARMONIC OSCILLATOR}

The one-dimensional Schrödinger equation describing the motion of a bounded particle has non-stationary solutions in the form of non-spreading wave packets only in the case of the linear harmonic oscillator. Each of such packets has the same shape as the wave function in a stationary state, but it performs harmonic oscillations with the ground frequency and with an arbitrary amplitude (s. formula (55), where $\psi_{n}$ denotes the wave function in a stationary state). The mean energy of the particle in such a non-stationary state is equal to the sum of the stationary state energy and the classical energy of the oscillations (s. formula (57)). The coefficients of the expansion (58) into the stationary states are expressed by the formulae $(60)-(62)$. 